\title{
Carnets
}

Revue électronique d'études françaises de l'APEF

Deuxième série - 13 | 2018

Corps, rythmes et voix : en/jeux littéraires et artistiques

\section{La multitude murmurante}

Retrouver les voix du mouvant et du temps

Avec Extrêmes et lumineux, de Christophe Manon (Lagrasse, Verdier, 2015)

Frédérique Cosnier-Laffage

\section{(2) OpenEdition}

1 Journals

Édition électronique

URL : http://journals.openedition.org/carnets/2658

DOI : $10.4000 /$ carnets. 2658

ISSN : 1646-7698

Éditeur

APEF

Référence électronique

Frédérique Cosnier-Laffage, "La multitude murmurante », Carnets [En ligne], Deuxième série -

13 | 2018, mis en ligne le 31 mai 2018, consulté le 20 avril 2019. URL : http://journals.openedition.org/ carnets/2658; DOI : 10.4000/carnets.2658

Ce document a été généré automatiquement le 20 avril 2019.

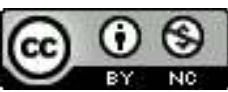

Carnets est mis à disposition selon les termes de la licence Creative Commons - Atribution - Pas d'utilisation commerciale 4.0 International. 


\title{
La multitude murmurante
}

\author{
Retrouver les voix du mouvant et du temps \\ Avec Extrêmes et lumineux, de Christophe Manon (Lagrasse, Verdier, \\ 2015)
}

\section{Frédérique Cosnier-Laffage}

« Le rythme est une subjectivation du temps, que

le langage retient du corps »

(Meschonnic, 1982:655)

\section{Saisissement}

1 Il s'agirait de partir d'une voix. Celle que j'ai entendue lors de la première lecture publique de Christophe Manon à laquelle j'ai assisté. Partir de cette voix, c'est partir d'un saisissement. Il lisait un extrait du poème $A u$ Nord $d u$ futur et il y eut un contraste inouï. D'une part la tranquillité d'une voix ténue, presque fragile, faite de ruptures, d'interruptions, de décrochages. D'autre part, l'ampleur d'un continu, sorte d'entêtement, de ténacité faite de relances, si tenace que cette lecture avait quelque chose de violent, en même temps que d'extrêmement calme. Lorsque je l'ai entendu lire un extrait d'Extrêmes et lumineux, qui va nous occuper ici, le même saisissement m'a gagnée. La tenue de ce double mouvement, entre décrochages et relance, est souvent soulignée dans les textes critiques qui évoquent la poétique de Manon à partir de sa voix, comme le fait par exemple Guénaël Boutouillet, parlant d'un « effet extrêmement singulier, un appel, une coupe brutale" côtoyant une "nécessité de relance», le tout formant une «fertile ambiguïté $»^{1}$.

2 Or, au-delà de cette ambivalence, il y eut pour moi comme une intimité directe, une familiarité avec un rythme, non définissable a priori, qui me fit immédiatement penser aux mots de Henri Meschonnic citant Eikhenbaum, qui écoutait lui-même Gogol en train de lire Le Manteau. Meschonnic retient que la lecture de Gogol « donne l'impression d'un 'mètre connu', sans être métrique » (Meschonnic, 1982 : 281), et que Gogol semble avoir la diction de son écriture. Le saisissement serait donc loin d'être anecdotique, et nous renseignerait sur la nature même de ce qu'est le rythme, ou du moins de ce qui advient 
lorsque l'on est en sa présence. Il y aurait comme la reconnaissance paradoxale d'un inconnu, et la sensation d'une indissociabilité entre diction et écriture. Comme si la diction était le corps physique de l'écriture, ou comme si la voix du poème était bien cette «activité » d'un " corps-langage », dont parle Serge Martin, ce qui reste du corps dans l'écriture (Martin, 2017 : 46).

Il semble que le poème ${ }^{2}$ de Christophe Manon, Extrême et lumineux, témoigne d'un pouvoir du langage par sa force singulière à inventer et tenir une forme de vie continue, alors même que son apparence fragmentaire pourrait nous faire penser à un discontinu du langage. En nous appuyant sur le concept meschonnicien de rythme, comme « organisation du mouvement de la parole dans le langage » (Bourlet, M. \& Gishoma, C., $2007: 2)^{3}$, nous verrons comment la voix du sujet-poème nous donne à lire sa propre écoute du multiple, puis comment elle donne forme à une écriture de la démesure, avant de nous demander si finalement cette démesure ne serait pas la recherche du présent des corps, voire du corps du présent.

\section{Voix du poème : une écoute du multiple}

4 L'oralité du texte apparaît d'emblée sous le jour de la fragmentation, donc du multiple, au risque du discontinu. Il est vrai que l'on est frappé par la multiplicité des époques et des lieux convoqués, dans un apparent désordre : du XvIII ${ }^{\mathrm{e}}$ siècle italien d'une petite troupe de saltimbanques, aux $\mathrm{xx}^{\mathrm{e}}$ et $\mathrm{xxI}^{\mathrm{e}}$ siècles des guerres, des paysages ruraux et urbains, des bars et des néons de parkings souterrains. De même, autre marque de multiplicité, on est en présence non seulement d'êtres humains, mais aussi d'un grand nombre de figures de la faune et de la flore, ainsi que de fantômes, spectres, créatures s'apparentant à des divinités mythiques voire archaïques. Comment la voix de ce poème nous fait-elle entendre ce multiple?

\section{Sonorité générale}

5 Extrêmes et lumineux est un texte très dense, composé de 70 fragments, s'ouvrant in medias res par un mot coupé, dont on n'a que la fin, «-oir» («- oir puis blanc puis noir ») et s'achevant sur la première lettre d'un mot, « $\mathrm{n}$ ». D'emblée, la fin est le début, et inversement, la boucle du temps se forme en brisant sa linéarité. Les fragments semblent juxtaposés, s'enchainant par ces mots coupés entre deux syllabes, sauf le dernier, sans numéro, sans lien narratif explicite autre qu'un système d'échos entre motifs et personnages récurrents. Ce sont bien les déboîtements successifs qui retiennent l'attention à première vue, même si au final le texte ne forme qu'une seule et unique phrase. Ruptures et continuité sont les principes qui sous-tendent l'architecture du texte. De fait, nous sommes face à un texte qui écoute une pluralité de voix à l'intérieur d'un même flux de matière, qui s'interrompent, s'entrecoupent, chacune semblant pousser l'autre pour faire sa place, ou bien on pourrait dire que c'est en s'accrochant l'une à l'autre qu'elles témoignent d'une érotique forte de son pouvoir d'engendrement, de projection, d'élan sans cesse relancé.

6 Pluralité et érotique de voix, ou plutôt de sans-voix. Le texte nous met en effet en présence des membres d'une famille, «multitude murmurante» (p.110) ${ }^{4}$, comédiens de fortune venus d'Italie, paysans rugueux et taiseux parmi lesquels un enfant a grandi, et dont le texte s'attache à faire vivre les grains de voix et tessitures. Comme le remarque 
une lectrice libraire de la librairie Charybde dans sa note de lecture ${ }^{5}$, l'évocation des " terres inhospitalières et des destins anonymes et ingrats " n'est pas sans rappeler les Vies minuscules de Pierre Michon. On pense bien évidemment également aussi à Claude Simon, notamment à Histoire, par cet entrelacs de diverses strates temporelles et mémorielles, par l'écriture du collage et de la diffraction. Or si les voix se précipitent les unes après les autres, c'est à leur écoute que nous sommes conviés par le dit du texte, celui-ci n'étant pas autre chose que l'écriture de cette écoute, attentive, obsédée, voire effarée. L'attention portée à la présence des voix est parfois signalée et vécue comme une véritable injonction à écouter. Au fragment $15^{6}$, le garçon se dit « forcé » à « écouter cette mélopée » du monologue de "la vieille femme » qui se livre à la narration de la partie italienne de l'histoire familiale, famille elle-même décrite comme « engeance volubile ». Dans son demi-sommeil, il est sensible à la consonance de patronymes, perdu « parmi des prénoms à consonance étrangère ", "pratiquement hypnotisé, ne percevant qu'un «murmure monotone qui se confond avec la plainte du vent». C'est en effet par ce murmure de la femme que le garçon a accès aux «méandres généalogiques » de sa propre histoire, les mots engendrant des formes physiques nimbées de merveilleux :

ces mots évoquant dans l'imagination du garçon un monde fantastique et marginal, plein de mystère - les images en noir et blanc de la télé éclairant la pièce par intermittence d'une lueur vaguement immatérielle, animant les ombres, les grandissant parfois démesurément en un théâtre d'illusion primitif, mouvant et trouble, pareilles à des êtres préhistoriques sur les parois d'une caverne sortis d'un rêve atavique (...) (p.41).

7 Le motif de la caverne et de la préhistoire marque depuis le tout premier chapitre la quête d'une histoire élargie bien au-delà des contours familiaux, pour venir frôler la dimension mythologique. Cela se lit dans la dénotation de la phrase, mais aussi dans son rythme, marqué par la reprise de la consonne $[\mathrm{m}]$ qui joue le rôle d'un véritable lien sonore entre différents motifs : vocalité du murmure, associé au mystère, au primitif, associé à la présence des images, et à l'évocation de l'«imagination». Celle-ci est d'ailleurs immédiatement liée à la démesure ("démesurément») et conduit au surgissement de la vie («animant»). On le voit, le rythme du texte nous place d'emblée en situation de lecture-écoute d'un acte créateur fondateur et démiurgique lié au murmure de la voix.

8 L'association de la voix à un élément naturel, évoquée dans ce fragment 15 par le murmure « qui se confond avec la plainte du vent ", se retrouve au fragment 66 , cette fois dans la voix d'un vieil homme, «s'exprimant d'une voix tonitruante et cependant avec parcimonie, dans une langue à peine sortie de sa gangue et qui donne le sentiment de charrier des monceaux de glaise et de boue, de rouler des brouettes de pierrailles et de gravats, ponctuant chacune de ses phrases de terribles jurons» (p.176). Le pouvoir créateur de la voix physique est encore évoqué ici sous les auspices du primitif, de l'archaïque, par le motif de la glaise et de la boue, associant toujours l'ancrage sociologique du tableau de famille à la dimension mythologique, de même qu'au fragment 27 le garçon entend les « voix retentissantes dans la cuisine », voix familiales aux

intonations et quelques mots surnageant tels des icebergs dans un océan sonore étranger, semblables en cet instant à des êtres chimériques, surnaturels, vaguement fabuleux et anachroniques, non pas des monstres, mais des dieux païens, brutaux et sanguins, ou encore des ogres, des géants, ou plutôt des figures sans âge. (pp. 61-62)

Mentionnons encore « le vieil homme », surpris au fragment 45 " passant le plus clair de son temps dans un monde parallèle et peuplé de revenants et de fantômes avec lesquels il 
entretient un dialogue ininterrompu ». Au-delà de l'univers familial, les voix des compagnons de bar sont également évoquées, avec leur «brouhaha » (p.46) ou encore le «flux incontrôlable » des «bavardages » (p.60) qui coule comme l'alcool des liqueurs et autres GET 27, au fragment 26.

Le texte semble faire ici " sonorité générale », pour reprendre l'expression de Péguy citée par Serge Martin, et c'est comme si l'auteur vivait «dans un affleurement perpétuel de textes [...] une masse énorme, (et non pas seulement des pensées), [où] des mondes veulent à chaque instant passer par la pointe de sa plume» (Martin, 2017: 92-93). La parole, par un processus de sédimentation mémorielle et de surgissement, a force d'action et déclenche à son tour le langage, constitue la voix plurielle de ce texte qui semble écrire une infinie réponse à la question de Walter Benjamin mise en exergue du livre : «N'est-ce pas autour de nous-mêmes que plane un peu de l'air respiré jadis par les défunts? N'est-ce pas la voix de nos amis que hante parfois un écho des voix de ceux qui nous ont précédés sur terre? $»^{7}$

\section{Pouvoir plastique des voix multiples}

11 Si le mécanisme mémoriel est souvent évoqué sous la forme d'un phénomène éminemment visuel, par l'évocation de flashs de lumière (le «lumineux » du titre), ou du phénomène de la révélation photographique - le narrateur se donnant tous les attributs de l'enquêteur face à une masse considérable d'archives picturales -, le flux vocal du texte, par cette multitude murmurante que nous venons d'évoquer, a bel et bien pouvoir d'engendrement d'une matière verbale. Le narrateur se présente d'ailleurs souvent dans un geste de façonnage sans cesse renouvelé. Ainsi, il est, page 81 «invité, encouragé, exhorté à modifier les faits, à en altérer la nature et le déroulement par la puissance fictionnelle du langage, par sa capacité à produire des énoncés de façon autonome ». Incorporant les voix du souvenir, la voix d'écriture fait naître une réalité, peut-être plus réelle encore que celle du vécu.

Ce phénomène est mis en abyme par exemple au fragment 43 , lorsque le narrateur lit une lettre reçue d'une ancienne amante, après que les douleurs «sont devenues une part irréductible d'eux-mêmes, aussi chère et précieuse que leurs plus extrêmes et lumineux instants » (pp. 103-104), et que les caractères de la lettre "semblent se déformer pour esquisser peu à peu les traits du visage depuis longtemps oublié de celle qui les a tracés ", réalisant par là un phénomène d'hypotypose, avant de «se dissoudre dans l'évocation de lointains souvenirs qui refluent soudain à la surface et envahissent sa mémoire, l'emplissant d'un flot d'images » (pp. 104-105). La voix de la lettre effectue ainsi un double mouvement, en plusieurs dimensions: elle crée, voire recrée un corps dans l'espace extérieur, sous les traits du visage de l'aimée, et elle effectue un mouvement vertical, souvent évoqué dans le livre, celui du reflux vers la surface, avant de se prolonger dans le mouvement horizontal du flot. La parole est donc infiniment mouvante, comme les souvenirs sont «mouvants, oscillants et instables» (p.140), et ce, sous des modalités multiples. Elle crée physiquement ce qu'elle évoque, à mesure qu'elle le dit.

Ce pouvoir de création physique de la voix de mémoire, constituant la matière même de la voix d'écriture, donne également au texte sa plasticité à un niveau macro-textuel. L'enquête menée par le narrateur à la recherche des traces de son passé, ou d'une histoire encore plus vaste, le mène à agencer entre eux des éléments de formes diverses qui constituent autant d'oralités singulières : fragments de mémoires du père (qui dialoguent 
souvent avec des photos retrouvées, annotées et commentées), cartes postales, extraits d'articles trouvés sur internet, annonce de vente aux enchères, bribes de paroles entendues, de monologues intérieurs du narrateur, de paroles rapportées des personnages, extraits de lettre comme nous venons de le voir. La variété des typographies, l'inscription des italiques ou des blancs, la disposition des fragments coupés au début et à la fin au beau milieu des mots donnent à voir une multiplicité mouvante et un foisonnement constituant ce que nous pourrions appeler un murmure choral et plastique.

\section{Mouvement dans le langage}

Cette mobilité extrême du texte, cette multiplicité des voix et l'aveu même du narrateur d'une nécessité de devoir tout reprendre, comme s'il fallait repartir du début, pourraient faire penser à une impossibilité du langage. Johan Faerber, dans un article pour Diacritik, écrit que, selon Manon,

l'écriture ne sert de rien. Elle est morte. Elle ne s'écrit plus. Pour Manon, l'écriture surgit comme le grand fantôme des hommes. Rien ne s'écrit qui ne se brise immédiatement : ainsi de toutes les phrases retranscrites qui sont déchirées d'ellesmêmes, comme autant de trouées, de manques, d'effondrements qui ne les rendent pas au sens, qui laissent la vision en suspension comme si chaque phrase ne faisait voir que sa propre impossibilité à dire. (Faerber, 2015 ${ }^{8}$ )

Le critique s'appuie pour cela sur certains aveux d'impuissance du narrateur aux prises avec un langage défectueux, par exemple lorsqu'il doute du pouvoir des mots, comme s'ils "permettaient d'insuffler une quelconque force, comme s'ils étaient investis d'un supposé pouvoir d'évocation alors qu'ils ne sont que les reflets fantomatiques d'euxmêmes, pâle troupeau égaré dans la lande du sensible» (Manon, 2015 : 109). Et Johan Faerber d'en conclure que «le langage ne [s'impose] plus comme l'idiome même des hommes", ou que «la littérature s'impose comme la grande analphabète des sentiments", ou encore que Manon "veut chercher la littérature ailleurs que dans la littérature, veut dès lors trouver la vision hors des mots ", que «l'image elle-même a lieu après l'image comme l'écriture a lieu bien après le langage, et la littérature bien après l'écriture ", puisque la littérature « est livrée à ce qui sort du discours, à ce qui fait oublier la tyrannie du langage » (Faerber, 2015).

Si l'on ne peut être que d'accord avec ce que Johan Faerber suggère d'un débordement, lorsqu'il souligne avec justesse que « chez Manon, l'image est portée par une énergie qui excède le langage : elle va vers la grande hypotypose des choses (...) comme si, chez Manon, l'image quittait la page et venait à flotter entre le langage et nous », nous ne pouvons qu'émettre des réserves sur cette idée permanente d'une écriture si puissante qu'elle en deviendrait un hors langage, permettant à «la Littérature d'atteindre à son Dehors inouï» et poser cette question : quel pourrait bien être ce «Dehors inouï»? Il nous semble en effet que c'est bien à l'intérieur du langage qu'il faut « ouïr » le pouvoir de sa voix. Si en effet, Manon paraît " trouver la vision hors des mots ", il ne la trouve peutêtre pas dans la littérature, mais plutôt en-dehors d'une littérature, celle qui ne serait pas la sienne. C'est peut-être et le langage et la littérature qu'il ne faut pas réduire à une approche sémiotique, celle du signe, du mot. Nous avons en effet depuis bien longtemps les outils théoriques pour penser, par le concept meschonnicien du rythme, que le langage est, s'il y a littérature, bien au-delà du signe : cet élan créateur, inconnu de luimême, qui invente dans et par le langage toujours un autre langage, c'est-à-dire du sujet, 
et le poème d'un sujet d'écriture. Il faut rappeler que «Tournures, rythmes, le langage tout entier d'une œuvre est l'activité d'un système, sa formation. Il n'a pas lieu dans la langue : la langue a lieu en lui. La littérature, de ce point de vue n'est qu'une spécification du fait qu'il n'y a pas, concrètement, de la langue: il n'y a que des discours... » (Meschonnic, 1982: 85) Ce que Johan Faerber veut sans doute souligner, lorsqu'il dit que la voix de Manon a lieu hors du langage ou " après », c'est que cette œuvre ne se crée pas dans la langue, mais qu'une langue, la voix du sujet poème Manon, a lieu dans ce « corpslangage » (Martin, $2017:$ 17) qu'est l'œuvre, au-delà de toute essentialisation de «la» littérature. C'est «le poème qui fait le poète, pas le poète qui fait le poème" (Meschonnic, 2005 : p. 257).

17 Il s'agirait dès lors de montrer comment le texte, alors que son dit déclare souvent une impuissance, fait autre chose par son dire, presque à mots, et non à corps, défendant.

\section{Rassembler les voix : vers le rythme de la démesure}

\section{Un phrasé synoral}

On peut repérer dans le texte au moins cinq passages constituant des sortes d'extraits d'art poétique insérés dans la narration: aux fragments 35 (pp. 79-82), 44 (pp. 107, 109-110), 48 (pp. 121-122), 54 (pp. 139-140, 142), 62 (pp. 162). Page 109, la voix d'écriture revient sur sa peine à composer le texte : «puis tout cela assemblé, compilé, distribué, disposé avec une tendresse maladroite dans une composition hasardeuse et aléatoire ». Or si le geste volontaire peut s'avérer hasardeux, ce que l'on entend au final est loin d'être dénué de liens et l'on constate dans certains enchainements de fragments des correspondances qui semblent plutôt tenir d'une nécessité, même si rétrospective. Ainsi des fragments 35,36 et 37 . Comme nous venons de le dire, le fragment 35 contient une première pause d'écoute dans le texte, où la voix revient sur sa propre dynamique et la commente :
-nant inlassablement les mêmes passages, corrigeant, amendant, modifiant, ajustant, ajoutant, supprimant, essayant dans un geste obstiné et presque vengeur d'épuiser la possibilité d'expression d'un souvenir, d'une réminiscence, d'un visage, d'une image, d'une sensation, d'émotions, de joies ou de tristesses (pp. 79-80)

Il faut noter l'irruption de cette idée de vengeance associée à celle du travail d'écriture et le caractérisant presque ( un geste obstiné et presque vengeur»), après une suite d'éléments plus techniques (" corrigeant, amendant, modifiant, ajustant, supprimant »). Or le fragment suivant, 36, contient en discours direct les paroles rapportées d'une femme qui pourrait bien être la mère, assénant à son fils une «formule que le garçon ressent comme une imprécation funeste et terrible à son encontre, un sortilège aberrant, un anathème irrévocable et vengeur " (p.82): «Tu ne feras pas toujours ce que tu veux dans la vie " (idem). N'est-ce pas contre cette vengeance de la mère, de ce «type de femmes » agissant « avec une espèce d'atavisme " voulant faire «payer à son tour son lot d'amertume et de déconvenues » (p.83) que se dresse en fait le geste lui-même vengeur de l'écriture qui vient juste d'être évoqué dans le fragment précédent? Où l'on assiste à une sorte de genèse de l'acte d'écrire, lorsque la voix reprend, toujours fragment 36 , avec "quelque chose dans son cœur se débattant tout de même furieusement, s'insurgeant, se révoltant, se promettant à l'avenir de s'efforcer malgré tout de contredire par tous les moyens dont il dispose cette sentence portée tel un oracle sur sa destinée», le 
«furieusement» et «par tous les moyens» ne pouvant que faire écho de manière saisissante à la longue suite de geste de composition évoqués quelques pages plus haut, et mis en actes tout au long du livre. On pourrait même lire le fragment qui vient encore plus bas, 37, et qui évoque le corps mort d'un vieil homme, et serait le résultat de cet acte de révolte, si ce n'est comme le meurtre du père, du moins comme la mise à mort définitive de l'oracle familial funeste, dont la voix d'écriture sort finalement victorieuse par le fait de faire œuvre.

Il semble donc que des relations de motivation sous-tendent la succession apparemment disparate des fragments, et définissent un geste jouant autant de la diffraction que du rassemblement, évoquant par là ce que Meschonnic appelle, en parlant de la voix physique, une puissance "synorale» (Meschonnic, 1982: 292) (mot calqué sur le mot "synoptique ») mais correspondant ici à la voix d'écriture. La voix, physique comme écrite, serait un " événement du phrasé », « acte syntaxique de liaison et de sens », « une rhétorique, une diction et un éros de l'écriture » (expressions de Jean-Pierre Martin citées par Serge Martin dans Martin, $2017: 71 »)$, en même temps qu'un acte de résistance et de révolte contre ce que d'autres voix auraient voulu pour elle.

21 Le plus saisissant de cet éros révolté réside bien sûr dans ce qui se joue au cœur des phrases, dans le déclenchement de la "mécanique verbale», évoquée par la voix ellemême à la page 142 .

\section{Dire le fracas : une écriture de la démesure}

Tout se passe comme si le rythme réalisait ce geste perpétuellement recommencé de ramasser le multiple et fabriquait sa propre matière en ne reconnaissant plus aucune limite, en démultipliant à l'infini tous les possibles, en s'emparant d'un "fracas vertigineux et indomptable de mots, d'images, de paroles, d'odeurs, de couleurs, de sensations, d'émotions confuses et indiscernables » (Manon, 2015 : 107). Il ne s'agit pas pour la voix de dompter ce fracas, de le traduire, de le reproduire tel qu'il se présente à la conscience mais bel et bien de le faire, dans et par le rythme du langage. De faire œuvre de vie, sans mesure, sans limite, avec tous les éléments par lesquels la vie surgit.

Par exemple, le fragment 47 évoque un moment de traite dans une étable. Ce souvenir se crée dans la démesure d'un "théâtre fantastique ", mettant en scène une vieille femme $\mathrm{au}$ « visage osseux de sorcière qui semble venu du fond des âges, accablé du poids de mille années ». Tout le texte s'écoule dans un rythme qui dit lui-même l'amplification sensorielle et créative sous de multiples formes. Ainsi, l'enfant observe

les gestes de la vieille femme (ou sorcière ou prêtresse donc) avec une attention non pas réservée, mais consternée, accablée, navrée, chagrine, outragée pour ainsi dire, l'odeur âcre et entêtante d'excréments, de lait chaud et de bétail faisant frissonner les narines en provoquant un haut-le-cœur qu'il peine à réprimer, esquissant alors un geste machinal de la main pour écarter le manège oppressant des insectes qui s'agglutinent sur son visage, ses joues, ses paupières, ses lèvres surtout, ayant la sensation d'être sur le point d'en avaler et d'être submergé, ne comprenant pas pourquoi cette archaïque intimité, cette profonde connivence pour laquelle il ne trouve même pas de nom, cette primitive familiarité entre la vieille femme et toute cette viande violette ce sang palpitant ces humeurs cette bave les entrailles dans leurs circonvolutions les muscles s'agitant sur leurs articulations les organes qui s'ébranlent lourdement les os puissants des cuisses et les tendons noyés dans la chair avec des viscères et des sabots et des cornes et des touffes de poils, pourquoi tout cela à la fois réalité et rêve l'émeut, le trouble, le bouleverse, le transperce de 
part en part au point de lui donner envie de s'enfuir à toutes jambes, de disparaître

et d'effacer à jamais cette image de sa mé (p.119)

24 On peut lire ici de mille manières cette écriture de la démesure. Par l'accumulation descriptive sans cesse relancée entre virgules, puis sans virgule, comme si la démultiplication des focalisations corporelles avalait les marques de séparation syntaxique (" son visage ", « ses joues ", « ses paupières ", « ses lèvres », / « cette viande »

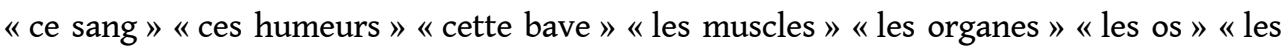
cuisses »), par les alternatives proposées ("femme ou sorcière ou prêtresse »), la

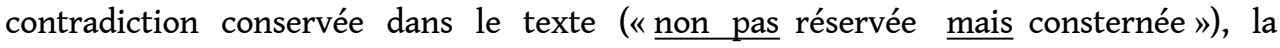
progression par accumulation de participes passés et d'adjectifs ("accablée, navrée, chagrine ») et par tâtonnement d'écoute (le "pour ainsi dire »), l'enchaînement par association des sens, (visuels avec l'image de la femme, olfactifs avec les évocations d'odeurs), débouchant sur l'évocation de cette conséquence physique qu'est la nausée et l'envie de fuir, bientôt suivie de la coupure véritable du fragment. On voit ici ce que le langage fait au corps, une exaspération des sens par le pouvoir d'activation de la parole, et ce que le corps fait au langage, une amplification quasiment infinie du déploiement rythmique de la voix. Et c'est sans parler, encore, du débordement de signifiance porté par le réseau consonantique $d u[r]$ se diffusant entre les corps humains et animaux et les reliant, de l'odeur animale jusqu'au désir de fuite du garçon: dans "consternée ", " navrée », " chagrine », « outragée », « dire ", « odeur », " âcre », " excrément », « frissonner ", « narine », " paupières », « lèvres », « entrailles », « cornes »... jusqu'au verbe «s'enfuir »... L'écriture se fait ici voix de corps, de sensations, toujours agissantes, vérifiant ce que remarque Serge Martin : que c'est comme "'forme-sujet' que le poème invente une interpénétration des formes de vie et des formes de langage, où la forme de vie fait langage et la forme de langage fait vie » (Martin, 2015 : 52).

25 A force d'amplification et de démesure, on pourrait se demander ce que la voix atteint finalement, et où nous porte cette voix de révolte. S'il ne s'agit pas de saisir le réel, geste dont le narrateur se défend à maintes reprises, quelle est donc la force ultime du langage poétique qui se déploie dans cette voix, emportant ainsi le multiple dans son murmure obstiné ?

\section{Rythme désir : dire le présent des voix et le corps du présent}

Si le geste d'écriture est ici acte de révolte, c'est que, encore bien au-delà des résonances dont nous venons de parler, cet acte semble bien devenir écriture du temps lui-même, et devenir par la texture physique du texte une fabrique du présent.

\section{Saisir : «Le passé fait corps avec le présent » (Bergson, 1934 :} 173)

Selon le philosophe Bergson, nous manquons la nature du temps lorsque nous pensons par notre intelligence que celui-ci est fait d'une succession d'instants discontinus. Nous croyons avoir affaire à des moments successifs, alors que la vraie nature du temps est la durée, qui peut être saisie dès lors que l'on écoute notre conscience par l'intuition. On perçoit alors que le temps est fait d'une «continuité indivisible de changement » 
(Bergson, 1934 : 166), le changement, le mouvement et la mobilité étant des mots-clés de la philosophie bergsonienne ${ }^{9}$. Or ce mouvement, ce changement, sont indivisibles au sens où, comme quand on perçoit une mélodie on ne peut percevoir des moments séparés de cette mélodie, de même dans l'écoulement du temps, il n'y a que du changement ininterrompu ${ }^{10}$.

Cette conception a une grande conséquence sur les relations entre passé et présent. S'il y a indivisibilité du changement et de la durée, il y a nécessairement «conservation du passé dans le présent ». Le présent n'est pas en opposition avec le passé mais étroitement liée à l'idée d'un continu, et c'est ainsi que "le passé fait corps avec le présent" (Bergson, 1934: 173, pour les deux citations précédentes). Bergson est celui qui veut redonner au présent son épaisseur.

On pourrait dire que c'est exactement ce que parvient à réaliser la voix d'écriture d' Extrêmes et lumineux, par sa puissance d'énonciation. En effet, elle semble bien être, selon les mots de Jacques Ancet sur l'énonciation poétique cités par Serge Martin, « le devenirprésent d'un sujet, d'un langage et d'un monde, inséparablement» (Martin, $2017: 121$ ). C'est ce que nous allons tenter d'examiner maintenant.

Le texte n'explique pas le sentiment d'une nécessité d'écrire par autre chose, précisément, que par le désir de « donner son épaisseur » au temps :

Quelle nécessité d'ajouter d'autres mots aux mots, quel impératif? [...] peut-être le rêve démesuré, inaccessible et présomptueux de tenter de saisir d'infimes éclats de vie et de ne pas les laisser s'éteindre, soufflant dessus comme sur braises, non pas de dire le monde, car la chose est résolument impossible, mais de le célébrer, non pas de retrouver le temps perdu mais de lui donner son épaisseur, sa mesure en quelque sorte » (Manon, $2015: 110)$

Dès lors, le rêve d'écrire ne peut qu'être associé à un acte, que l'on reconnaît à de nombreuses reprises dans le texte, celui de «saisir ». Saisir un corps (« s'emparer de son corps luxuriant, le saisir, le manger le triturer le pétrir » p. 55), chercher quels souvenirs fantomatiques saisir ("quels fils saisir, pour mener où, faire surgir quels fantômes, quelles ombres » p. 80), saisir la permanence des vies («comme si, par une opération magique, il était possible de franchir les distances temporelles et de rejoindre les vies depuis longtemps révolues saisies dans cette éphémère permanence » pp. 92-93), ou saisir des éclats de vie («le rêve démesuré, inaccessible et présomptueux de tenter de saisir d'infimes éclats de vie et ne pas les laisser s'éteindre " page 110 / "le réel étant précisément discontinu, formé d'éléments juxtaposés sans raison (...), d'autant plus difficiles à saisir qu'ils surgissent de façon sans cesse imprévue » p. 142) : la matérialité du texte naît de ce désir de saisissement. Et ce n'est pas une surprise si ce désir se trouve associé à celui de saisir des voix, celle d'une famille régulièrement évoquées, par exemple un monologue entendu qui devient, par métaphore, une pierre que l'on pourrait prendre entre ses mains, car cette pierre a le poids du temps: "désir désespéré non pas de comprendre, mais de saisir pour de bon entre ses petites mains d'enfant comme une grosse pierre rugueuse et pesante du poids des siècles cet incompréhensible et lancinant monologue " (p.41). Où l'on découvre finalement que saisir des voix par l'écriture, c'est saisir du temps, c'est le rendre palpable, dans sa suspension: son présent. N'est-ce pas l'intuition de ce phénomène qui provoquait la sensation avec laquelle nous avons ouvert cette recherche, le saisissement dont nous sommes pris lorsque nous écoutons une lecture de Christophe Manon? 


\section{La force d'un désir : « réanimer l'imperceptible lueur d'une présence » (Manon, $2015:$ 80)} présent, par exemple dans la belle image «l'haleine du temps» (p.109), que ce texte exerce de nouveau son pouvoir de rassemblent, au-delà du fragmentaire, en réunissant les extrêmes du temps : «l'éphémère permanence » (p.93) «l'éphémère durée » (p.102) de toute vie humaine. La voix ne doute pas, finalement, que les mots, et plus que les mots bien sûr, tout le rythme du texte, ont "le pouvoir d'insuffler une quelconque force » (p.109) pour nous permettre d'affronter ce temps qui passe, « comme un fragile rempart contre le néant pour compenser la pauvreté du matériau ou peut-être comme pour ralentir l'écoulement du temps qui s'accélère et s'emballe » (p.122). On constate donc que l'écriture acquiert ce pouvoir, par le rythme, de déjouer le rythme apparemment implacable et discontinu du temps, pour mieux révéler celui de la durée. En cela, il semble que la question qui ouvre le livre, demandant qui peut bien être «l'artiste dément à l'orgueil insensé » ayant réalisé les «œuvres monumentales», "peintures pariétales ultra-contemporaines » (p.9) que sont les surfaces de couleurs observées dans un parking sous-terrain, pourrait être une question s'appliquant à l'artiste capable de faire résonner une telle voix : quel sujet de poème est donc capable de ce geste ? La réponse serait qu'il s'agit bien de cette voix du poème-Manon, voix d'orgueil et de démesure, voix des sansvoix fondues dans une seule, défiant l'oubli et l'impuissance du langage en même temps qu'il les dit, pour célébrer au contraire l'épiphanie ${ }^{11}$ de la parole poétique, sans cesse renouvelée, et naissante, dans le corps et le rythme de chaque page.

\section{Pour relancer les écoutes}

Nous partageons le regret exprimé par Meschonnic lorsqu'il dit dans une partie de Critique du rythme consacrée à Bergson que « là où la pensée traditionnelle du temps, de la durée, a été contestée, modifiée, vers une pensée du continu, le langage est resté dans la théorie du signe, qui est discontinu » (Meschonnic, 1982 : 176). En effet, Bergson conçoit la langue dans son aspect fixé, donc incapable, précisément, de rendre compte de la durée et du changement. Le moment où il rate la rencontre avec ce qui se passe dans le poème, et donc dans le rythme, est celui où il semble bien avoir l'intuition qu'il se passe quelque chose d'absolument singulier par le rythme, mais où il le rejette hors du langage, un peu comme ce que fait à sa manière Johan Faerber. Meschonnic dit bien que Bergson aboutit 
même à un positionnement de l'ordre de "l'impossible, de l'incompréhensible ", qui le « conduit à concevoir l'art comme un contre-langage » (Meschonnic, 1982 : 179). En effet, par exemple quand Bergson veut faire l'éloge de l'écrivain, il dit que «l'art de l'écrivain constitue surtout à nous faire oublier qu'il emploie des mots » (Bergson, 1912, repris dans Meschonnic, $\left.1982: 181^{12}\right)$.

On voit ce qu'aurait pu être une continuation de la philosophie bergsonnienne du temps jusqu'à une philosophie du langage. À la fin de sa conférence sur «La perception du passé », Bergson dit que l'art " dilate notre perception », " enrichit notre présent, mais il ne nous fait guère dépasser le présent ». Et il prétend que c'est par la philosophie que " nous pouvons nous habituer à ne jamais isoler le présent du passé qu'il traîne avec lui » (Bergson, 1934: 175, pour les dernière citations). Pour conclure, si Bergson n'a pas poursuivi sa quête jusqu'à une philosophie du langage, on peut du moins entendre tout ce qui, dans son approche du monde par sa philosophie du temps, pourrait se dire du pouvoir du rythme et s'appliquer à la poétique telle qu'elle se met en mouvement dans Extrêmes et lumineux. En effet, grâce à la philosophie vue par Bergson, comme grâce la poétique mise en actes par Manon, on pourrait dire que toutes choses acquièrent

comme une quatrième dimension qui permet aux perceptions antérieures de rester solidaires des perceptions actuelles, et à l'avenir immédiat lui-même de se dessiner en partie dans le présent. La réalité n'apparaît plus alors à l'état statique, dans sa manière d'être ; elle s'affirme dynamiquement, dans la continuité et la variabilité de sa tendance. Ce qu'il y avait d'immobile et de glacé dans notre perception se réchauffe et se met en mouvement. Tout s'anime autour de nous, tout se revivifie en nous. Un grand élan emporte les êtres et les choses. Par lui nous nous sentons soulevés, entraînés portés. Nous vivons davantage » (Bergson, 1934 : 176)

\section{BIBLIOGRAPHIE}

BERGSON, Henri (1934). La Pensée et le mouvant. Paris : PUF, coll. Quadrige, 2013.

BOURLET, M. \& Gishoma, C. (2007). « Des voix dans la poésie : Entretien avec Henri Meschonnic ». Études littéraires africaines, $n^{\circ} 24$, pp. 4-11 [consulté le 30.12.2017], <URL :https://www.erudit.org/ fr/revues/ela/2007-n24-ela02388/1035338ar/resume/>

FAERBER, Johan, (2015). « Christophe Manon (Extrêmes et lumineux) : Le monde sur le bout de la langue », [consulté le 16.10.2017] <URL :https://diacritik.com/2015/10/27/christophe-manonextremes-et-lumineux-le-monde-sur-le-bout-de-la-langue/>

MANON, Christophe (2015). Extrêmes et lumineux. Lagrasse : Verdier.

MARTIN, Serge (2017). Voix et relation, Une poétique de l'art littéraire où tout se rattache. Taulignan : Marie Delarbre, collection "Théories".

MESCHONNIC, Henri (1982). Critique du rythme, Anthropologie historique du langage. Lagrasse : Verdier. MESCHONNIC, Henri (2005). « Oui, qu'appelle-t-on penser ? », in Gérard Dessons, Serge Martin et Pascal Michon (éds), Henri Meschonnic, la pensée et le poème. Colloque de Cerisy 12-19 juillet 2003, Editions IN PRESS, pp. 251-266. 


\section{NOTES}

1. Voir la note de lecture consacrée à Extrêmes et lumineux sur le blog de Guénaël Boutouillet

(14.08.2015), [disponible le 14.01.2018], <URL: https:// materiaucomposite.wordpress.com/2015/08/14/christophe-manon-extremes-et-lumineuxeditions-verdier-aout-2015/ > ou le même article repris sur le site remue.net qui propose plusieurs états antérieurs du travail <URL : http://remue.net/spip.php?article7720 >

2. Bien qu'il soit désigné comme roman, je qualifie le texte de Manon de poème, selon la définition du poème de Meschonnic : «l'invention d'une forme de vie par une forme de langage et l'invention d'une forme de langage par une forme de vie » (Meschonnic, $2005: 257$ ).

3. Dans Critique du rythme, Meschonnic donne cette définition précise et détaillée du rythme: «l'organisation des marques par lesquelles les signifiants, linguistiques et extralinguistiques (dans le cas de la communication orale surtout) produisent une sémantique spécifique, distincte du sens lexical, et que j'appelle la signifiance : c'est-à-dire les valeurs, propres à un discours et à un seul. Ces marques peuvent se situer à tous les 'niveaux' du langage : accentuelles, prosodiques, lexicales, syntaxiques. (...) Contre la réduction courante du 'sens' au lexical, la signifiance est de tout le discours, elle est dans chaque consonne, dans chaque voyelle qui, en tant que paradigme et que syntagmatique, dégage des séries ». (Meschonnic, $1982: 217$ ).

4. Lorsque le nom de l'auteur et la date ne sont pas précisés, les numéros de pages renvoient toujours au texte de Christophe Manon, Extrêmes et lumineux. Voir bibliographie.

5. Note du 27.09.2015, [disponible le 13.01.2018] <URL: https:// charybde2.wordpress.com/2015/09/27/note-de-lecture-extremes-et-lumineux-christophemanon/>

6. Les fragments ne sont pas numérotés dans le texte, comme nous l'avons dit, c'est nous qui les numérotons pour faciliter le repérage.

7. Cette citation n'est pas référencée dans le livre de Manon, mais provient de «Sur le concept d'histoire ", dans Walter Benjamin, Ecrits français, 1991, Paris, Gallimard, collection Folio, p. 433.

8. L'article n'étant pas paginé ni les lignes numérotées, les citations ne peuvent être précisément référencées mais se trouvent au fil du texte. Voir références en bibliographie.

9. Voir par exemple ces expressions dans Bergson, 1934, «Le mouvement est la réalité même » p. 159, « la réalité est la mobilité même » p. 168.

10. Sur la mélodie comme durée indivisible voir toujours Bergson, $1934: 166$.

11. Je me permets d'employer ce mot utilisé par l'auteur lui-même dans un échange que nous avons eu à propos de son texte.

12. Conférence de Bergson, «L'âme et le corps », 1912.

\section{RÉSUMÉS}

Lorsque l'on écoute une lecture performée par Christophe Manon, on est saisi par le caractère inédit d'un tissu sonore fait de ruptures, où le murmure le dispute à un flux obstiné que rien ne semble pouvoir arrêter. Dans le poème du continu corps-voix qu'est Extrêmes et lumineux, nous sommes plongés au cœur d'une narration in medias res, immédiatement portés par un rythme marqué par l'hétérogénéité (narrations interrompues, pluralité de discours rapportés, morceaux 
de lettres, de listes, etc.) en même temps que par l'unité d'une forme-sens ramassant le multiple. C'est à partir de ce constat que nous verrons comment le mouvement de cette voix, à l'écoute de son propre désir de saisir quelque chose du temps, effectue le geste démesuré de rassembler des voix, et ne se propose rien moins que de rendre le présent palpable par une physique et une plastique vocales, faisant par là écho, en poétique, à l'intuition philosophique du temps selon Bergson, comme mouvement et durée sans cesse réinventés.

When we listen to a reading by Christophe Manon, we are struck by the originality of a "sound fabric" made of ruptures, where the murmur is a constant stream of music that nothing seems to be able to stop. In the poem of the continuous body-voice that is Extrêmes et lumineux, we are plunged into the heart of a narrative in medias res, immediately carried by a rhythm that is marked by heterogeneity (interrupted narrations, plurality of reported speeches, pieces of letters, lists, etc.), and marked by the unity of a form-sense gathering the multiple. It is from this observation that we will see how the movement of this voice, listening to its own desire to grasp something of time, carries out the inordinate gesture of gathering voices, and proposes nothing less than to make the present palpable by a vocal both physical and plastic, echoing in this way through poetics, the philosophical intuition of time according to Bergson, as a movement and duration constantly reinvented.

INDEX

Mots-clés : mouvement, corps, langage, désir, présent, Bergson (Henri)

Keywords : movement, language, body, desire, present, Bergson (Henri)

\section{AUTEUR}

\section{FRÉDÉRIQUE COSNIER-LAFFAGE}

Centre de Linguistique Appliquée, Université de Franche-Comté. Doctorante Paris 3 Sorbonne, UMR THALIM,

frederique.cosnier-laffage[at]univ-fcomte.fr 